\title{
ЧИСЛЕННОЕ МОДЕЛИРОВАНИЕ ГОРЕНИЯ ВОДОРОДО-ВОЗДУШНЫХ ГАЗОВЫХ СМЕСЕЙ
}

\section{NUMERICAL SIMULATION OF GORENJE OF HYDROGEN-AIR GAS MIXTURES}

S. Martyushov

Summary. The applicability of various sets of reactions and coefficient values in the Arrhenius formulas for calculating the ignition of hydrogen-air mixtures is verified. The approbation of the kinetic model of a branching chain reaction for calculating the initial stage of gorenje and detonation of such mixtures is considered. Test one-dimensional calculations of the ignition of mixtures in a small closed heat-insulated region are carried out.

Keywords: gorenje hydrogen-air mixtures, branching chain reaction, rigid systems of differential equations.

\author{
Мартюшов Сергей Николаевич \\ Д.ф.-м.н., профессор, ФГБОУ ВО «Московский \\ авиационный институт (национальный \\ исследовательский университет)» \\ martyush@mail.ru
}

Аннотация. Проведена проверка применимости различных наборов реакций и значений коэффициентов в формулах Аррениуса для расчета воспламенения водородо-воздушных смесей. Рассмотрена апробация кинетической модели ветвящейся цепной реакции для расчета начальной стадии горения и детонации таких смесей. Проведены тестовые одномерные расчеты воспламенения смесей в малой замкнутой теплоизолированной области.

Ключевые слова: горение водородо-воздушных смесей, ветвящаяся цепная реакция, жесткие системы дифференциальных уравнений.

ной реакции в водородовоздушной смеси, описанный первоначально в работах Н.Н. Семенова [5].

\section{Кинетическая модель}

в первую очередь, с задачей конструирования детонационных двигателей, работающих на водородо-воздушной смеси. Перспективными результатами в этой области являются различные конструкции гипотетических двигателей, в частности импульсных [1] и ротационных [2, 3]. В то же время необходимо констатировать, что задача по-прежнему далека от технического воплощения. Одним из направлений продвижения в этом направлении является совершенствование математических моделей и численных методов для расчета процессов воспламенения газовой смеси и перехода первоначального горения в устойчивую детонацию.

Первоначально многими авторами для численного моделирования использовалась модель упрощенной химической реакции, предложенной Левиным [1], которая позволила получить существенные результаты, например, в работах Фудживары [4]. Для более детального исследования, в том числе для аккуратного учета энергетического баланса, в настоящее время, используются модели, основанные на учете более или менее полной системе химических реакций $(9,16,26$ и более реакций) протекающих в водородовоздушной смеси в процессе инициации воспламенения и перехода горения в детонацию. С точки зрения кинетики процесс перехода к детонации можно рассматривать как переход от горения и теплового взрыва к ветвящейся цеп-
Для моделирования реакций горения в смесях водород-воздух обычно используется система обыкновенных дифференциальных уравнений, основанная на гипотезе Аррениуса о скоростях химических реакций. Уравнения, описывающие химические реакции, можно представить в виде

$$
\sum_{i=1}^{n} \alpha_{i j} A_{i}=\sum_{i=1}^{n} \beta_{i j} B_{i}, j=1, \ldots, M
$$

где $M, n$-число реакций и компонент смеси, соответственно, $A_{i}, B_{i}$ стехиометрические коэффициенты прямой и обратной реакций. Скорости изменения концентраций компонент смеси определяются в соответствии с гипотезой Аррениуса.

$$
\begin{aligned}
& f_{i}=\frac{d c_{i}}{d t}=\sum_{j=1}^{M}\left(\beta_{i j}-\alpha_{i j}\right) w_{j}(\stackrel{r}{c}, T) \\
& w_{j}(c, T)=k_{f}(T) \prod_{i=1}^{n} c_{i}^{\alpha_{i j}}-k_{b}(T) \prod_{i=1}^{n} c_{i}^{\beta_{i j}} \\
& k_{f}=A_{f} T^{l} \exp \left(-E_{f} / R T\right)
\end{aligned}
$$

Для обеспечения не убывания энтропии и корректного расчета тепловыделения коэффициенты обратных реакций рассчитывались с помощью константы равновесия: 
Таблица 1.

\begin{tabular}{|l|l|l|}
\hline$-\mathrm{H}_{2}+\mathrm{O}_{2}=2 \mathrm{OH}$ & $-\mathrm{H}_{2}+\mathrm{OH}=\mathrm{H}+\mathrm{H}_{2} \mathrm{O}$ & $-2 \mathrm{HO}_{2}=\mathrm{H}_{2} \mathrm{O}_{2}+\mathrm{O}_{2}$ \\
\hline$-\mathrm{H}+\mathrm{O}_{2}=\mathrm{O}+\mathrm{OH}$ & $-\mathrm{H}_{2}+\mathrm{O}=\mathrm{H}+\mathrm{OH}$ & $-\mathrm{HO}_{2}+\mathrm{M}=\mathrm{H}+\mathrm{O}_{2}+\mathrm{M}$ \\
\hline$-\mathrm{H}_{2}+\mathrm{M}=2 \mathrm{H}+\mathrm{M}$ & $\mathrm{H}_{2} \mathrm{O}_{2}+\mathrm{M}=2 \mathrm{OH}+\mathrm{M}$ & $\mathrm{OH}+\mathrm{H}_{2} \mathrm{O}=\mathrm{H}+\mathrm{H}_{2} \mathrm{O}_{2}$ \\
\hline
\end{tabular}

$$
k_{b} / k_{f}=K=\exp \left[\sum_{i=1}^{n}\left(\beta_{i j}-\alpha_{i j}\right)\left(\frac{G_{i}^{0}(T)}{R T}+\ln \frac{R T}{P_{0}}\right)\right]
$$

В многочисленных работах используются схемы окисления водорода с различным числом реакций и компонент смеси. Значения коэффициентов $k_{f, b}$, задающих скорости реакций в (4) значительно различаются в работах различных авторов [6-8]. В то же время результаты газодинамических расчетов горения и детонации иногда в решающей степени зависят от того, какой набор реакций и с какими значениями коэффициентов $k_{f, b}$, был выбран.

Одной из задач настоящего исследования была проверка применимости различных наборов реакций и значений коэффициентов в модели (2)-(4).

В качестве тестовой одномерной задачи решалась следующая задача: в некотором замкнутом объеме, первоначально заполненном стехиометрической смесью водород-воздух с атмосферными значениями газодинамических параметров, в начальный момент времени температура повышалась скачком до значений, при которых должна была начаться реакция горения (около $3 T_{\text {атм }}$ в градусах Кельвина).

Численный эксперимент состоял в данном случае в расчете термодинамических параметров газовой смеси в малом теплоизолированном объеме, при этом значения мольных концентраций газовой смеси рассчитывались при помощи решения жесткой системы обыкновенных дифференциальных уравнений по явному численному методу Гира и проводился до полного выгорания водородной компоненты или определению отсутствия возгорания в разумные пределы времени.

Термодинамические параметры находились последовательно из значений потенциала Гиббса:

$$
\begin{aligned}
& G\left(\rho, T, c_{i}\right)=\sum_{i=1}^{N} c_{i}\left[R T \ln \left(P c_{i} / P_{0} \sum_{i=1}^{N} c_{i}\right)+G_{i}^{0}(T)\right] \\
& H_{i}^{0}(T)=G_{i}^{0}(T)-T \frac{d G_{i}^{0}(T)}{d T} \\
& h=\sum c_{i} H_{i}^{0}(T), V=\left(R T \sum_{i=1}^{N} c_{i}\right) / P
\end{aligned}
$$

Рассматривалась 9-компонентная газовая смесь: $\mathrm{H}_{2}$, $\mathrm{O}_{2}, \mathrm{H}, \mathrm{O}, \mathrm{H}_{2} \mathrm{O}, \mathrm{OH}, \mathrm{HO}_{2}, \mathrm{H}_{2} \mathrm{O}_{2}, \mathrm{~N}_{2}$. При этом пренебре- галось наличие компонент $A r$ (1\% атмосферного воздуха, участие в виде катализатора в ряде промежуточных реакций) и $\mathrm{O}_{3}$ (соответствующие реакции с участием озона, вообще говоря, могут давать определенный вклад в конечную выделяемую энергию).

Начальный период реакции, период индукции, характеризуется накоплением в смеси радикалов $H, O$, $\mathrm{OH}$, (а также $\mathrm{HO}_{2}, \mathrm{H}_{2} \mathrm{O}_{2}$ )., концентрация которых последовательно возрастает и убывает, от $\mathrm{H}, \mathrm{O}$ к $\mathrm{HO}_{2}$, $\mathrm{H}_{2} \mathrm{O}_{2}$, (см. Рис. 2, А). Существенное уменьшение концентрации $\mathrm{H}_{2}$ и $\mathrm{O}_{2}$ и образование конечного продукта $\mathrm{H}_{2} \mathrm{O}$ происходит на второй стадии реакции. Скорость протекания обеих стадий существенно возрастает с увеличением начального скачка температуры и при малой величине такого скачка реакция не происходит за время счета или зависимость от времени убывания $\mathrm{H}_{2}$ и $\mathrm{O}_{2}$ и роста концентрации $\mathrm{H}_{2} \mathrm{O}$ имеет плавный характер, соответствующий медленному горению водорода (см. Рис. 1А).

Проведение расчетов с 9, 16 и 26 реакциями, с коэффициентами, выбранными в соответствии с [6], показало, что увеличение числа реакций не приводит к качественному изменению графиков концентрации компонент или скорости протекания процесса, поэтому для дальнейшего моделирования было решено ограничиться набором из 9 наиболее используемых различными авторами реакций.

В качестве базовых были приняты следующие реакции (таблица 1).

Использовались 2 набора коэффициентов в формулах(2) для реакций Таблицы 1: первый из [6] (для расчета медленного горения) и из [7] (для расчетов на основе теории ветвящейся цепной реакции.

Графики изменения концентраций составляющих смеси приведены на Рис. 1А превышение температуры $2.4 T_{\text {атм }}$ и Рис. 1, В для превышение температуры 2.6 $T_{\text {amм }}$ небольшое увеличение температуры качественно меняет скоростьпротекания реакции.

Графики изменения концентраций составляющих смеси при значении констант из [7] и превышение температуры $2.8 T_{\text {атм }}$ приведены на Рис. 2А. Для наглядности значения концентраций радикалов $\mathrm{O}, \mathrm{H}, \mathrm{OH}, \mathrm{HO}_{2}$, 


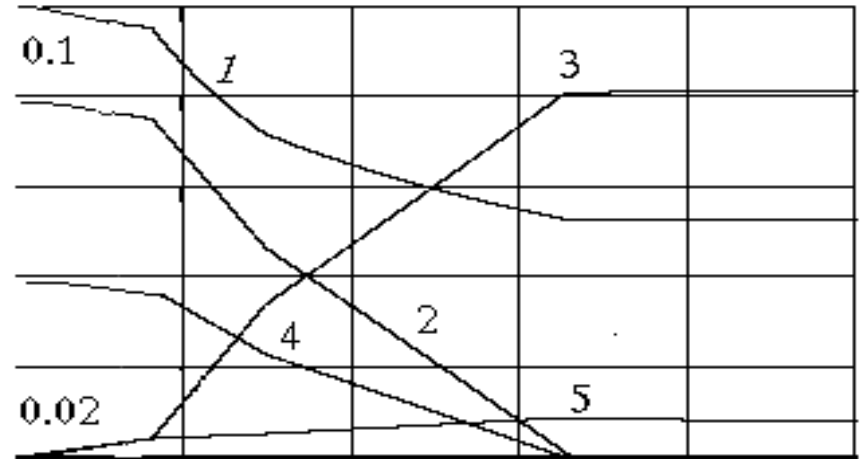

A

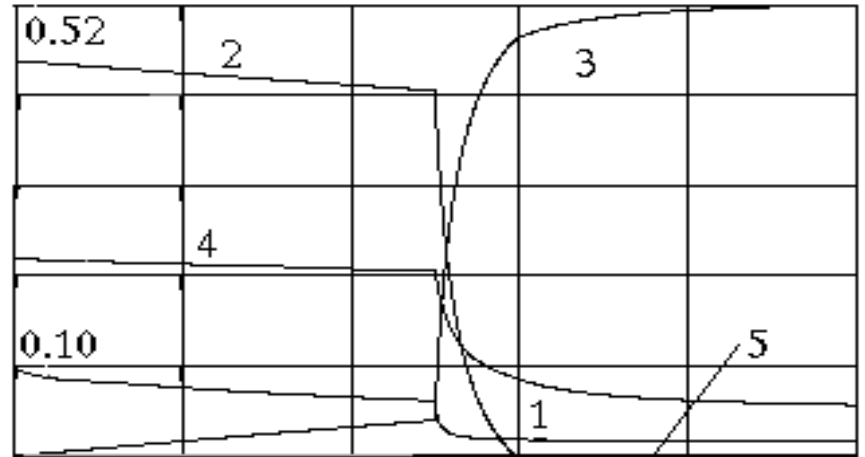

B

Рис. 1А. Графики массовой концентрации компонент смеси (использованы константы из [6]), В процессе медленного горения, линии: 1-1/Т - обратная температуре, 2 - H2, $3-\mathrm{H} 2 \mathrm{O}, 4-\mathrm{O} 2$, 5 - ОН в масштабе 105. Для Рис. 1А. превышение температуры 2.4 Татм, для Рис. 1В. превышение температуры 2.5 Татм, небольшое увеличение температуры качественно меняет вид реакции.

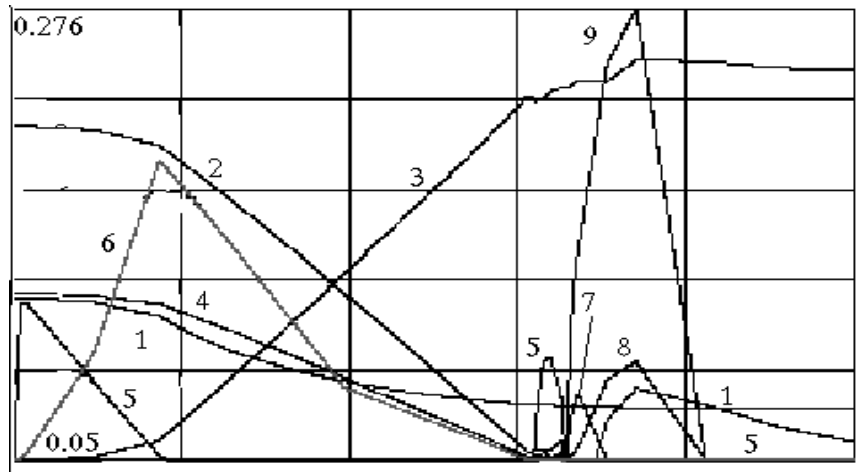

A

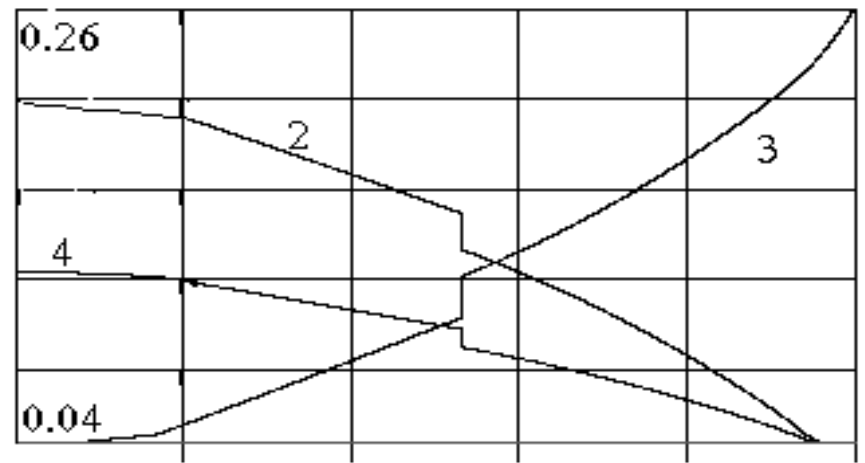

B

Рис. 2. Графики массовой концентрации компонент смеси (использованы константы из [2]), в процессе медленного горения, линии: 1-1/Т - обратная температуре, $2-\mathrm{H} 2,3-\mathrm{H} 2 \mathrm{O}, 4-\mathrm{O} 2,5$ $-\mathrm{OH}, 6-\mathrm{H}_{,}, 7-\mathrm{HO}_{2} 8-\mathrm{O}, 99-\mathrm{H} 2 \mathrm{O} 2$, компоненты 5-9 в масштабе 105.

$\mathrm{H}_{2} \mathrm{O}_{2}$ умножались на масштабные коэффициенты, составлявшие $10^{5}$.

Характерным свойством горения смеси $\mathrm{H}_{2}-\mathrm{O}_{2}$ является наряду с медленным горением (Рис. 1А) появление режима моментального взрыва, который происходит после иногда длительного периода индукции (Рис. 1В). В этот период происходит накопление в смеси радикалов $H, O$ и $O H$, при этом количество этих радикалов в смеси остается малым и происходит переход от одного радикала к другому (Рис. 2, величина компонент-радикалов умножается на коэффициент $10^{5}$ для наглядности).

Механизм этого взрыва - ветвящиеся цепные реакции радикалов предложен Н.Н. Семеновым [5]. Для построения модели ветвящейся цепной реакции в [7] использовались реакции (с соответствующими скоро- стями $k_{0}-k_{5}$ ). Рассматривалась следующая схема цепной реакции:

9. $\mathrm{H}_{2}+\mathrm{O}_{2}=2 \mathrm{OH}$ - возникновение цепи, скорость реакции (в соответствии с (3) $-W_{0}=k_{0}\left[H_{2}\right]$ $\left[\mathrm{O}_{2}\right]$.

1. $\mathrm{H}_{2}+\mathrm{OH}=\mathrm{H}+\mathrm{H}_{2} \mathrm{O}$ продолжение цепи, $W_{1}=k_{1}$ $\left[\mathrm{H}_{2}\right][\mathrm{OH}]$

2. $\mathrm{H}+\mathrm{O}_{2}=\mathrm{O}+\mathrm{OH}$ разветвление цепи, $W_{2}=k_{2}[\mathrm{H}]$ $\left[\mathrm{O}_{2}\right]$

3. $\mathrm{H}_{2}+\mathrm{O}=\mathrm{H}+\mathrm{OH}$ разветвление цепи, $W_{3}=k_{3}\left[\mathrm{H}_{2}\right]$ [O]

4. $H+$ cтенка $=0.5 H_{2}$ обрыв цепи на стенке, $W_{4}$ $=k_{4}[\mathrm{H}]$

5. $\mathrm{O}_{2}+\mathrm{H}+\mathrm{M}=\mathrm{HO}_{2}+\mathrm{M}$ обрыв цепи в объеме, $W_{5}=$ $k_{5}[\mathrm{H}]\left[\mathrm{O}_{2}\right]$,

где $k_{i}$ определяются по формуле (4). Скорости обратных реакций вычислялись по формуле (5). 
В соответствии с теорией ветвящихся цепных реакций в ходе процесса цепной реакции радикалы $H, O$, $O H$ многократно вступают в реакцию с остальными компонентами смеси, при этом их масса остается малой, они полностью расходуются в ходе реакции, поэтому к $O$, $O H$ применяется метод квазистационарных концентрации (скорость изменения их концентраций полагается равной нулю). Для компоненты $H$ рассматривается упрощенное дифференциальное уравнение с использованием скоростей реакции $W_{0}-W_{5}$, при этом скорость роста $H$ существенно превышает скорости изменения «медленных» компонент $\mathrm{H}_{2}, \mathrm{O}_{2}, \mathrm{H}_{2} \mathrm{O}$, поэтому это уравнение должно решаться в другом временном масштабе.

В соответствии [5,7] скорости изменения молярной концентрации $H, O, O H$, могут быть представлены следующим образом:

$$
\begin{aligned}
& d[H] / d t=W_{1}-W_{2}+W_{3}-W_{4}-W_{5 ;} \\
& 0=d[O] / d t=W_{2}-W_{3} ; \\
& 0=d[O H] / d t=2 V_{0}+2 W_{2}-W_{1} . \\
& \text { Откуда } W_{2}=W_{3} \text { и } W_{1}=2 V_{0}+2 W_{2} \\
& \text { Для }[H] \text { получаем: } \\
& d[H] / d t=2 V_{0}+2 W_{2}-W_{4}-W_{5}, \text { откуда } \\
& d[H] / d t=2 V_{0}+2 k_{2}[H]\left[O_{2}\right]-k_{4}[H]-k_{5}[H]\left[O_{2}\right] .
\end{aligned}
$$

Или, с учетом членов второго порядка по [H]:

$$
\begin{gathered}
d[\mathrm{H}] / d t=2 k_{0}\left[\mathrm{H}_{2}\right]\left[\mathrm{O}_{2}\right]+\Phi[\mathrm{H}]-\mathrm{K}[\mathrm{H}]^{2} \\
\text { где } \left.\Phi=2 k_{2}\left[\mathrm{O}_{2}\right]-k_{4}-k_{5}\left[\mathrm{O}_{2}\right]\right), K= \\
2\left(k_{2}{ }^{2} k_{2} / k_{1} k_{3}\right)\left(\left[\mathrm{O}_{2}\right] /\left[\mathrm{H}_{2}\right]\right)^{2}
\end{gathered}
$$

При $\Phi>0[H]$ - решение (5) быстро возрастает, что соответствует ветвящейся цепной реакции. Молярные концентрации квазистационарных компонент [O] и $[\mathrm{OH}]$ находятся из алгебраических соотношений:

$$
\begin{aligned}
& {[\mathrm{O}]=[\mathrm{H}]\left(\mathrm{k}_{2}\left[\mathrm{O}_{2}\right]\right) /\left(k_{3}\left[\mathrm{H}_{2}\right]\right)} \\
& {[\mathrm{OH}]=2\left[\mathrm{O}_{2}\right]\left(k_{0}+k_{2}[\mathrm{H}] /\left[\mathrm{H}_{2}\right]\right) / \mathrm{K} 1 .}
\end{aligned}
$$

Остальные компоненты смеси получаются из решения оставшихся уравнений системы (2), с константами реакций, соответствующих [5].

Переход к расчету по алгоритму ветвящейся цепной реакции естественно применять при выполнении условия $\Phi>0$. Критерий прекращения расчета по алгоритму ветвящейся цепной реакции не очевиден. В настоящей работе расчеты по этому алгоритму прекращались по мере выгорания кислорода, а именно при уменьшении абсолютной величины скорости его убывания. В рассматриваемой модели полагалось $W_{4}=0$, то есть влияние стенок считалось для рассматриваемого атмосферного давления (вблизи верхнего предела по давлению «полуострова воспламенения») пренебрежимо малым.

Графики изменения концентраций составляющих смеси при значении констант из [7] и превышении температуры $3.2 T_{\text {атм }}$ с использованием алгоритма расчета ветвящейся цепной реакции, приведены на Рис. 2, В.

\section{ЛИТЕРАТУРА}

1. Левин В.А., Марков В.В., Осинкин С.Ф. Инициирование детонации в водородовоздушной смеси взрывом сферического заряда ТНТ. Физика горения и взрыва. 1995, Т. 31, № 2, С. 91-95

2. Levin V.A., Nechaev Y.N., Tarasov A.I., Control of detonation processes. Ed.G. Roy. Moscow, Elex-KM Publishers 197-201, 2000.

3. Zhdan SA. Bykovskii F.A. Vedernikov F.F. Mathematical modeling of a rotating detonation wave in a hydrogen-oxygen mixture. Combustion. Explosion and Shock Waves. 2007, 43(4): 449-459.

4. Таки С., Фудзивара Т. Численный анализ двумерных нестационарных детонационных волн// Ракетная техника и космонавтика 1978. Т. 16, № 1. с. 9398.

5. Семенов Н.Н. Самовоспламенение и цепные реакции. Успехи химии Т. 36 № 1 с. 3-22, 1967.

6. Ibragimova L.B., Smechov G.D., Shatalov 0.P. Recommended Rate Constants of Chemical reactions in an H2-02 Gas Mixture with Electronucally Excited Species 02, 0, OH Involved. Institute of Mechanics of Lomonosov Moscow State University, Moscow, 2003.

7. Денисов Е.Т., Саркисов 0.М., Лихтенштейн Г.И. Химическая кинетика. М. Химия, 200, 568с. 